\title{
Measuring Dirac CP-violating phase with intermediate energy beta beam facility
}

\author{
P. Bakhti ${ }^{\mathrm{a}}$, Y. Farzan ${ }^{\mathrm{b}}$ \\ School of Physics, Institute for Research in Fundamental Sciences (IPM), P.O. Box 19395-5531, Tehran, Iran
}

Received: 9 July 2013 / Accepted: 5 February 2014 / Published online: 25 February 2014

(C) The Author(s) 2014. This article is published with open access at Springerlink.com

\begin{abstract}
Taking the established nonzero value of $\theta_{13}$, we study the possibility of extracting the Dirac CP-violating phase by a beta beam facility with a boost factor $100<$ $\gamma<450$. We compare the performance of different setups with different baselines, boost factors, and detector technologies. We find that an antineutrino beam from ${ }^{6} \mathrm{He}$ decay with a baseline of $L=1300 \mathrm{~km}$ has a very promising CP-discovery potential using a 500 kton water Cherenkov detector. Fortunately this baseline corresponds to the distance between FermiLAB to Sanford underground research facility in South Dakota.
\end{abstract}

\section{Introduction}

The developments in neutrino physics in recent 15 years have been overwhelmingly fast. Nonzero neutrino mass has been established and five out of nine neutrino mass parameters have been measured with remarkable precision. In 2012, finally, the relatively small mixing angle, $\theta_{13}$ was measured [1-3]. This nonzero value of $\theta_{13}$ opens up the possibility of having $\mathrm{CP}$-violating effects in the neutrino oscillations; i.e., $P\left(v_{\alpha} \rightarrow v_{\beta}\right) \neq P\left(\bar{v}_{\alpha} \rightarrow \bar{v}_{\beta}\right)$. With this nonzero value of $\theta_{13}$, the quest for measuring the Dirac CP-violating phase, $\delta_{D}$, has been gathering momentum. A well-studied way to extract $\delta_{D}$ is the precision measurement and comparison of $P\left(v_{\mu} \rightarrow v_{\mathrm{e}}\right)$ and $P\left(\bar{v}_{\mu} \rightarrow \bar{v}_{\mathrm{e}}\right)$ by superbeam and neutrino factory facilities [4]. However, this is not the only way. In fact by studying the energy dependence of just one appearance mode e.g., $P\left(v_{\mu} \rightarrow v_{\mathrm{e}}\right)$, the value of $\delta_{C P}$ can be extracted [5]. In [6], a novel method for extracting $\delta_{D}$ (or more precisely $\cos \delta_{D}$ ) was suggested that was based on reconstructing the unitary triangle in the lepton sector. The idea of reconstructing the unitary triangle in the lepton sector has been later on studied in [7-13].

\footnotetext{
a e-mail: pouya_bakhti@ipm.ir

be-mail: yasaman@theory.ipm.ac.ir
}

Recently, beta beam facilities producing $v_{\mathrm{e}}$ or $\bar{\nu}_{\mathrm{e}}$ beams from the decay of relativistic ions [14-21] have been proposed and studied as an alternative machine to establish $\mathrm{CP}$ violation in the neutrino sector. Most studies were, however, performed before the measurement of $\theta_{13}$, with a focus on the CP-discovery reach for values of $\theta_{13}$ much smaller than the measured one [22]. References [23,24] show that using the $v_{\mathrm{e}}$ beam from ${ }^{18} \mathrm{Ne}$ decay with energies peaked around 1.5-2 GeV, information on $\delta_{D}$ can be extracted without need for an antineutrino beam. In this setup, the boost factor of the decaying ions is $\gamma=450$. In the present work, we shall consider a similar setup; however, with lower boost factors yielding neutrino energies below the 13-resonance energy in the mantle, which is about $6.5 \mathrm{GeV}$ [25]. For a detailed analysis of the matter effects see [26]. For a neutrino beam with a given energy in the range $400 \mathrm{MeV}<E_{v}<1.5 \mathrm{GeV}$, the oscillation probability can be approximately written as

$P_{e \mu} \simeq\left|c_{12}^{m} c_{23}\left(\mathrm{e}^{i \lambda_{2}}-\mathrm{e}^{i \lambda_{1}}\right)+s_{13}^{m} s_{23} \mathrm{e}^{-i \delta_{D}}\left(\mathrm{e}^{i \lambda_{3}}-\mathrm{e}^{i \lambda_{2}}\right)\right|^{2}$

where $c_{12}^{m} \ll 1$ is the cosine of the 12 -mixing angle in matter and $\lambda_{i}$ are the phases resulting from the propagation; i.e., for a constant density $\lambda_{i}=\left(m_{i}^{2}\right)$ eff $L /(2 E)$. For the antineutrino mode, a similar equation holds with $s_{12}^{m} \ll 1$ and

$P_{\bar{e} \bar{\mu}} \simeq\left|s_{12}^{m} c_{23}\left(\mathrm{e}^{i \lambda_{2}}-\mathrm{e}^{i \lambda_{1}}\right)+s_{13}^{m} s_{23} \mathrm{e}^{i \delta_{D}}\left(\mathrm{e}^{i \lambda_{3}}-\mathrm{e}^{i \lambda_{1}}\right)\right|^{2}$.

Notice that in the above formulas, the deviations of the values of $\theta_{23}$ and $\delta_{D}$ in matter from those in vacuum are neglected. These deviations are of order of $\Delta m_{12}^{2} / \Delta m_{13}^{2}$ [26]. As long as $\left|\lambda_{2}-\lambda_{1}\right| \sim 1$, the two terms in Eq. (1) as well as those in Eq. (2) are of the same order so the interference terms which are sensitive to $\delta_{D}$ are of order of the oscillation probabilities themselves. This means that the variation in the oscillation probabilities due to the change of $\delta_{D}$ within $(0 \pi)$ is of order of the oscillation probabilities, themselves. As a result for these energies and $\left|\lambda_{2}-\lambda_{1}\right| \sim 1$, even a moderate precision 
in the measurement of the probabilities will be enough to extract the value of $\delta_{D}$.

The flux at the detector scales as $\gamma^{2}$ and the scattering cross section of neutrinos increases by increasing the energy (i.e., increasing $\gamma$ ). As a result, for a given baseline, the statistics increases with $\gamma$. Based on this observation, most attention in recent years has been given to $\gamma>300$. However, one should bear in mind that for $\gamma<300$, there is the advantage of using very large water Cherenkov (WC) detectors. In this energy range, the neutrino interaction will be dominantly quasi-elastic and its scattering cross section is known with high precision.

In the literature, the $\mathrm{CP}$-discovery potential of a beta beam setup from CERN to Frejus with $\gamma<150$ and $L=130 \mathrm{~km}$ has been investigated [27]. Moreover, varying the values of $\gamma$ and baselines, it has been shown that for $150<\gamma<300$ with 500 kton WC detector [28-30] or iron calorimeter [31], there is a very good chance of CP-discovery. Reference [22] explores the $\theta_{13}-\delta_{D}$-discovery reach with a $300 \mathrm{kton} \mathrm{WC}$ and 50 kton LAr detector at Deep Underground Science and Engineering Laboratory (DUSEL), taking maximum boosts possible at Tevatron. Now that the value of $\theta_{13}$ is measured and found to be sizeable, reconsidering $\gamma<300$ setup is imperative. In vacuum, the dependence of the oscillation probability on $L$ and $\gamma$ would be through $L / \gamma$. However, for setups under consideration, because of the matter effects, the dependence on $L$ and $\gamma$ is more sophisticated so the dependence on $E$ and $L$ has to be investigated separately. In particular, while Ref. [28] focuses on $L / \gamma=2.6 \mathrm{~km}$, we have found that for $L / \gamma>2.6 \mathrm{~km}$, there is a very good discovery potential. The present paper is devoted to such a study.

In Sect. 2, we describe the inputs and how we carry out the analysis. We outline the characteristics of the beam and the detector as well as the sources of the background and the systematic errors. In Sect. 3, we present our results and analyze them. In Sect. 4, we summarize our conclusions and propose an optimal setup for the $\delta_{D}$ measurement.

\section{The inputs for our analysis}

Using the GloBES software [32,33], we investigate the CPdiscovery potential of a beta beam setup with various baselines and beam boost factors, $\gamma$. For the central values of the neutrino parameters, we have taken the latest values in Ref. [34]. The hierarchy can be determined by other experiments such as PINGU [35-41] or combining PINGU and DAYA Bay II results [42], so we assume that this hierarchy is known by the time that such a beta beam setup is ready. We study both normal and inverted hierarchies. T2K and Nova can also solve the octant degeneracy and determine whether $\theta_{23}<45^{\circ}$ or $\theta_{23}>45^{\circ}$ [43]. The data already excludes the $\theta_{23}>45^{\circ}$ solution at $1 \sigma \mathrm{CL}$. For the uncertainty of the mix- ing parameters, we take the values that will be achievable by forthcoming experiments. Namely, we take the following uncertainties: $0.4 \%$ for $\theta_{12}$ [44], $1.8 \%$ for $\theta_{13}$ [45], $2 \%$ for $\theta_{23}$ [46], $0.2 \%$ for $\Delta m_{12}^{2}$ [44] and $0.7 \%$ for $\Delta m_{13}^{2}$ [3540]. As predefined by GLoBES, we use the matter profiles in $[47,48]$. We consider $5 \%$ error for matter density. The uncertainties are treated by the so-called pull-method [32,33]. While the effects of uncertainty in matter density is more important for larger baselines, the uncertainties of neutrino parameters affect the results from smaller baselines more.

As the source of neutrino (antineutrino) beam, we take decays of ${ }^{18} \mathrm{Ne}\left({ }^{6} \mathrm{He}\right)$ :

${ }^{18} \mathrm{Ne} \rightarrow{ }^{18} \mathrm{~F}+\mathrm{e}^{+}+v_{\mathrm{e}}$

and

${ }^{6} \mathrm{He} \rightarrow{ }^{6} \mathrm{Li}+\mathrm{e}^{-}+\bar{v}_{\mathrm{e}}$.

The endpoint energies of these two decays are very close to each other: $E_{0}=3.4 \mathrm{MeV}$ for ${ }^{18} \mathrm{Ne}$ and $E_{0}=3.5 \mathrm{MeV}$ for ${ }^{6} \mathrm{He}$. As a result for equal $\gamma$, the energy spectrum of $\nu_{\mathrm{e}}$ and $\bar{\nu}_{\mathrm{e}}$ from their decays will be approximately similar. The pair of ${ }^{8} \mathrm{Li}$ and ${ }^{8} \mathrm{~B}$ isotopes have also been discussed in the literature as a potential source of the $v_{\mathrm{e}}$ and $\bar{\nu}_{\mathrm{e}}$ beams. In these cases, the endpoints are higher so as to have neutrino beams with energies $E_{v}<1.5 \mathrm{GeV}$, the values of $\gamma$ should be lower than in the case of ${ }^{18} \mathrm{Ne} /{ }^{6} \mathrm{He}$. On the other hand, the flux at the detector drops as $\gamma^{-2}$ so with the ${ }^{8} \mathrm{Li}$ and ${ }^{8} \mathrm{~B}$ isotopes, the number of decays should be larger to compensate for the $\gamma^{-2}$ suppression. We will not consider the ${ }^{8} \mathrm{Li} /{ }^{8} \mathrm{~B}$ isotopes in this paper and will focus on the ${ }^{18} \mathrm{Ne} /{ }^{6} \mathrm{He}$ pair. For the neutrino (antineutrino) mode, we take $2.2 \times 10^{18}\left(5.8 \times 10^{18}\right)$ decays of ${ }^{18} \mathrm{Ne}\left({ }^{6} \mathrm{He}\right)$ per year, which seems to be realistic [49]. The Tevatron accelerator can accelerate ${ }^{18} \mathrm{Ne}$ and ${ }^{6} \mathrm{He}$ up to boost factors 586 and 351, respectively.

While the disappearance probabilities (i.e., $P\left(v_{\mathrm{e}} \rightarrow v_{\mathrm{e}}\right)$ or $\left.P\left(\bar{v}_{\mathrm{e}} \rightarrow \bar{v}_{\mathrm{e}}\right)\right)$ are not sensitive to $\delta_{D}$, the appearance probabilities (i.e., $P\left(v_{\mathrm{e}} \rightarrow v_{\mu}\right)$ or $\left.P\left(\bar{v}_{\mathrm{e}} \rightarrow \bar{v}_{\mu}\right)\right)$ contain information on $\delta_{D}$. In our analysis, we, however, employ both appearance and disappearance modes. In principle, the disappearance mode can help to reduce the effect of uncertainty in other parameters but we have found that the effect of turning off the disappearance mode on the $\delta_{D}$ measurement is less than $1 \%$. To derive the value of $\delta_{D}$, the detector has to distinguish $v_{\mu}$ from $v_{\mathrm{e}}$. We focus on a $500 \mathrm{kton}$ water Cherenkov (WC) detector and compare its performance with a 50 kton totaly active scintillator detector (TASD).

In the energies of our interest with $\gamma<300$, the main interaction mode is Charged Current (CC) quasi-elastic mode with a non-negligible contribution from inelastic charged current interaction which produce one or more pions along with the charged lepton. In principle, the quasi-elastic CC events can be distinguished from the inelastic $\mathrm{CC}$ ones by counting the number of Cherenkov rings. However, to dis- 
tinguish the two interactions with a WC detector will be challenging. We take the signal to be composed of both quasi-elastic and inelastic charged current events and conservatively assume that the WC detector cannot distinguish between the two.

In the case of QE interaction by measuring the energy and the direction of the final charged lepton, the energy of the initial neutrino can be reconstructed up to an uncertainty of $0.085 \mathrm{GeV}$, caused by the Fermi motion of the nucleons inside the nucleus. However, in the inelastic interaction, a fraction of the initial energy is carried by pions, so the energy of the initial neutrino cannot be reconstructed by measuring the energy and the direction of the final lepton alone. A WC detector cannot measure the energy deposited in hadronic showers, so with this technique the reconstruction of the energy spectrum will be possible only for the $\mathrm{QE}$ interactions. Following the technique in $[28,50]$ we take an unknown normalization for $\mathrm{QE}$ events and use its spectrum as a basis for energy reconstruction. Of course, with this method energy reconstruction cannot be carried out on an event by event basis and information on the spectrum will only be statistical. TASD can measure the energy deposited in hadronic showers, too. As a result, energy reconstruction by TASD can be possible on an event by event basis.

As shown in [51], the background from atmospheric neutrinos can be neglected and the main source of background for both TASD and WC detectors are neutral current interactions of the beam neutrinos. In our analysis, for the cross sections of the quasi-elastic, inelastic and neutral current interactions we employ the results of $[52,53]$. Recently the MiniBooNE collaboration has measured the antineutrino cross section in the energy range of our interest [54] with remarkable precision. In the near future, the measurement of the cross section will become even more precise. Unless otherwise stated, we assume 4 years of data taking.

For the treatment of the efficiencies and backgrounds we implement the same methods as used in $[28,50]$. While for the purpose of this paper the methods used in $[28,50]$ are adequate, we would like to note that a more complete discussion of reconstruction of events in the large WC detectors can be found in [51]. To be more specific, similarly to [28] we assume the following characteristics for the WC detector performance. We take a signal efficiency of $55 \%$ for neutrinos and of $75 \%$ for antineutrinos. We take the uncertainty in the normalization of the total signal to be $2.5 \%$ but as we mentioned above, we take a free normalization for QE events. We assume a background rejection of $0.3 \%$ for neutrinos and $0.25 \%$ for antineutrinos. The normalization uncertainty of the background is taken to be $5 \%$. For both background and signal, the calibration error is 0.0001 . For the energy reconstruction of the background, we use the migration matrices tabulated for the GLoBES package $[55,56]$. We consider the energy range between 0.2 and $3 \mathrm{GeV}$ and divide it into 28 bins. The energy resolution for QE CC interactions is assumed to be of the form $0.085+0.05 \sqrt{E / \mathrm{GeV}} \mathrm{GeV}$ for both muon and electron neutrino detection. The first term originates from the Fermi motion of the nucleons inside nuclei and the second term reflects the error in measuring the energy of the final charged lepton [22].

As in Ref. [28], we assume the following features for TASD: A signal efficiency of $80 \%$ for $v_{\mu}$ and $\bar{v}_{\mu}$ and of $20 \%$ for $v_{\mathrm{e}}$ and $\bar{v}_{\mathrm{e}}$; background rejection of $0.1 \%$; a signal normalization uncertainty of $2.5 \%$; normalization uncertainty of $5 \%$; a calibration error of 0.0001 . The energy resolution is given by $0.03 \sqrt{E / \mathrm{GeV}} \mathrm{GeV}$ for muon (anti)neutrinos and $0.06 \sqrt{E / \mathrm{GeV}} \mathrm{GeV}$ for electron (anti)neutrinos. The energy range is taken to be $0.5-3.5 \mathrm{GeV}$ and is divided into 20 bins. We have studied the dependence of our results on the number of bins. It seems that the results do not change by increasing the number of bins to 30 .

\section{Results and the interpretation}

In Figs. 1 and 3, the vertical axis shows the precision with which $\delta_{D}=90^{\circ}$ can be determined at $1 \sigma \% \mathrm{CL}$. We take $\delta_{D}=90^{\circ}$ and define $\Delta \delta_{D}$ to be the range for which $\Delta \chi^{2}<1$. More precisely, $\Delta \delta_{D}$ is defined as the difference between maximum and minimum values of $\delta_{D}$ around $\delta_{D}=90^{\circ}$ for which $\Delta \chi^{2}=1$. From Fig. 1, we observe that the low energy setup with $\gamma=300$ and WC detector can outperform the setup with $\gamma=450$ and TASD detector for both normal and inverted hierarchies. The oscillatory behavior of the curves is driven by the 13-splitting and has a frequency given by $\sim \Delta m_{31}^{2} /(2 E)$. Such a behavior can be understood by the following consideration on Eqs. (1) and (2): While $\lambda_{2}-\lambda_{1}$ is driven by $\left(\Delta m_{21}^{2}\right)_{\mathrm{eff}}$ and slowly varies with $L, \lambda_{3}-\lambda_{2}$ is driven by $\left(\Delta m_{32}^{2}\right)_{\text {eff }}$ and varies rapidly. For the values of $L$ with $\lambda_{3}-\lambda_{2}=2 n \pi$, the sensitivity is lost. This consideration explains the oscillatory behavior of Fig. 1. Notice, however, that this consideration holds for a given $E_{v}$. If the energy spectrum is wide, the effect will smear out. In other words, if the number of energy bins from which information on $\delta_{D}$ can be deduced (i.e., the bins for which the number of events without oscillation is sizeable and the quasi-elastic interactions dominate) is relatively large, missing information in few of these bins for which $\left(\lambda_{3}-\lambda_{2}\right) \rightarrow 0$ will not affect much the precision in the determination of $\delta_{D}$. In the opposite case, when at all such bins $\left(\lambda_{3}-\lambda_{2}\right) \rightarrow 0$, the precision in $\delta_{D}$ will be dramatically deteriorated. Increasing the boost factor increases both the peak energy and the energy width. Thus, we expect for higher $\gamma$ that the oscillatory behavior is to be smeared out. Figure 1 confirms this expectation. In the case of antineutrinos, the information on $\delta_{D}$ can be deduced from a larger range of the spectrum mainly because of the shape of the spectrum at the source and the fact that for antineutrinos, 


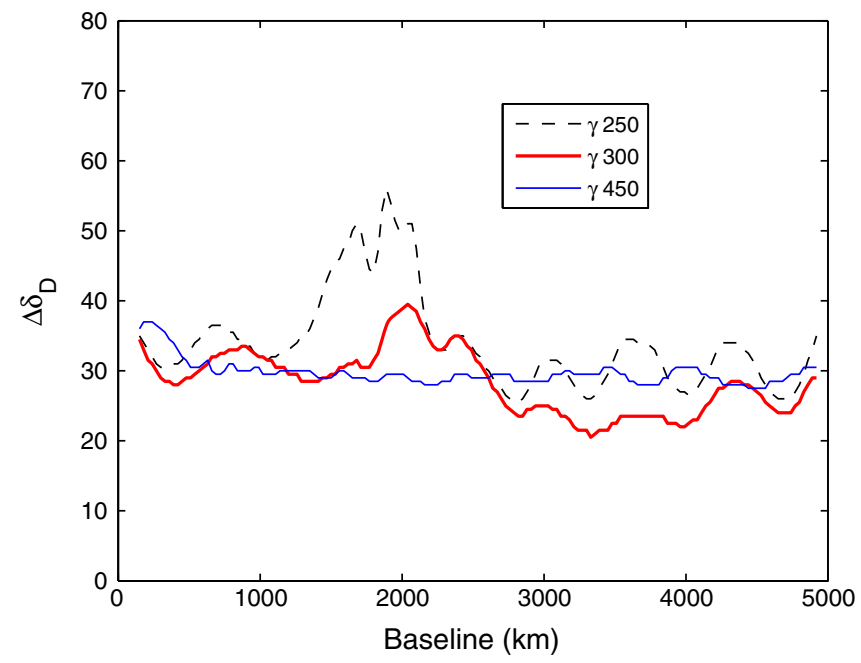

(a) Neutrino beam, normal hierarchy

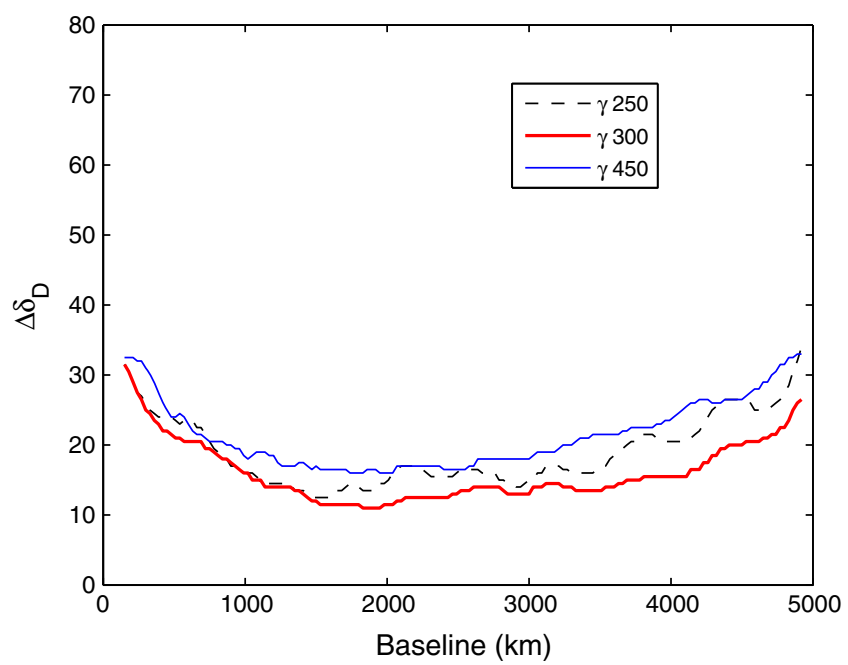

(c) Antineutrino beam, normal hierarchy

Fig. 1 Uncertainty within which $\delta_{D}=90^{\circ}$ can be measured at $1 \sigma \mathrm{CL}$ after 4 years of data taking versus baseline for different values of the boost factor. For $\gamma=450$, a 50 kton TASD detector and for lower $\gamma$, a 500 kton WC detector are assumed. In the upper (lower) panels, a neutrino (antineutrino) beam with $2.2 \times 10^{18}\left(5.8 \times 10^{18}\right)$ decays per

the QE interactions dominate over the inelastic interaction for a wider energy range compared to the case of neutrino $[52,53]$. As a result, the modulation driven by $\Delta m_{31}^{2}$ is less severe for antineutrinos. As seen in the lower panels of Fig. 1, the antineutrino beam with $\gamma=300$ and a WC detector can achieve an impressive precision of better than $20^{\circ}$ for baselines over $500 \mathrm{~km}$.

Figure 2 shows the fraction of the parameter $\delta_{D}$ for which $\mathrm{CP}$-violation can be established. From these figures, we also observe that setups with $\gamma<300$ and a 500 kton WC detector can outperform the setup with a 50 kton TASD detector and $\gamma=450$ for $L<2500 \mathrm{~km}$.

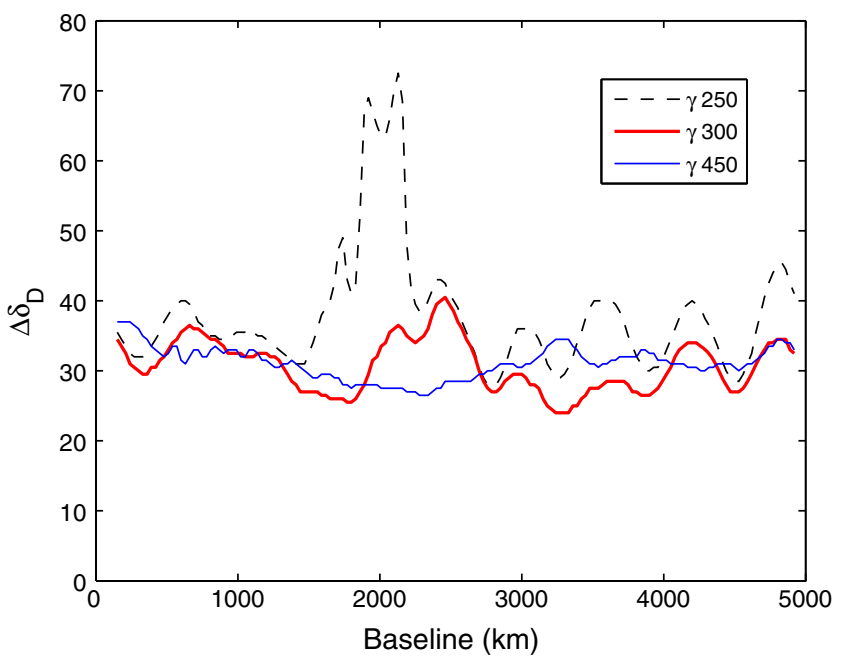

(b) Neutrino beam,i nverted hierarchy

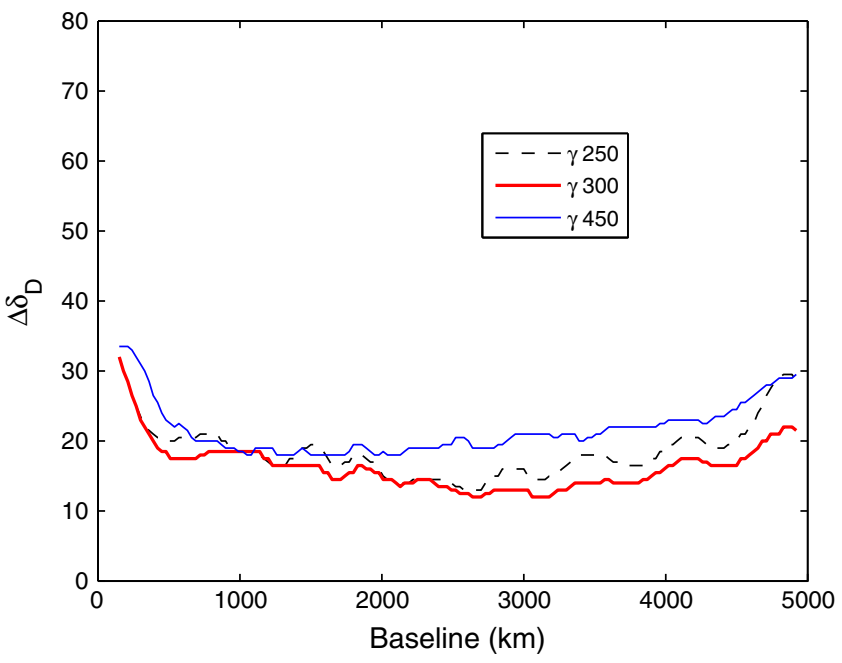

(d) Antineutrino beam, inverted hierarchy

year is assumed. In the left (right) panels, the hierarchy is taken to be normal (inverted). We have taken the true values for the left panels as $\Delta m_{31}^{2}=2.421 \times 10^{-3} \mathrm{eV}^{2}$ (normal hierarchy) and $\theta_{23}=41.4^{\circ}$ (first octant). For the right panels we have taken $\Delta m_{31}^{2}=-2.35 \times 10^{-3} \mathrm{eV}^{2}$ (inverted hierarchy) and the same mixing angles

Figures 3 and 4 compare the CP-discovery potential of a $v$ run with an $\bar{v}$ run and a mixed balanced run. For the antineutrino run the decay rate is taken to be about 2.6 times that of neutrinos to compensate for the low cross sections of antineutrinos. For $200 \mathrm{~km}<L<5000 \mathrm{~km}$, the antineutrino run seems to outperform both the neutrino run and the mixed run in the precision measurement of $\delta_{D}=90^{\circ}$. This result is at odds with the results of [28]. However, we should remember that Ref. [28] focuses on a specific value of $L / \gamma$. In this energy and baseline range, the sensitivity of the average $P\left(\bar{v}_{\mathrm{e}} \rightarrow \bar{v}_{\mu}\right)$ to $\delta$ is higher than that of the average $P\left(v_{\mathrm{e}} \rightarrow v_{\mu}\right)$. 


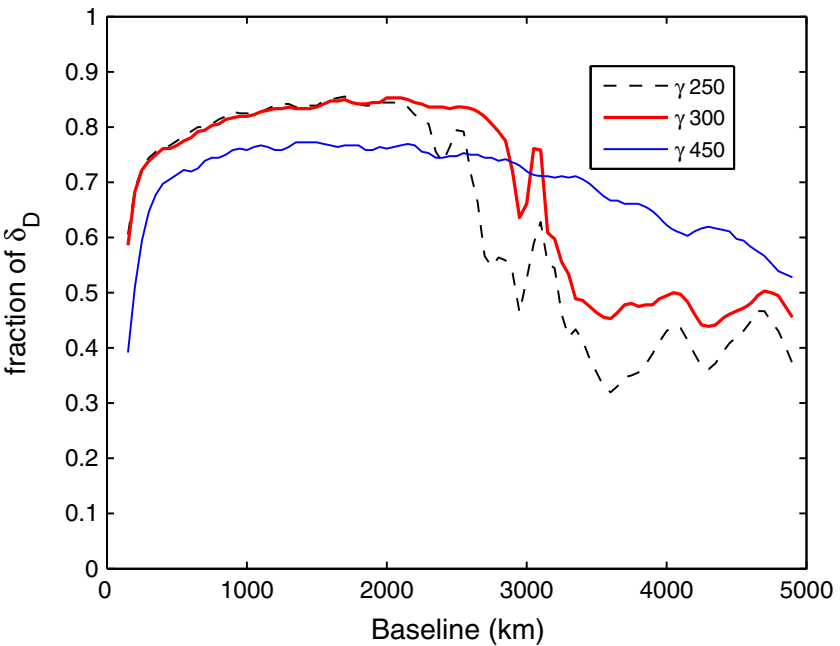

(a) Neutrino beam, normal hierarchy

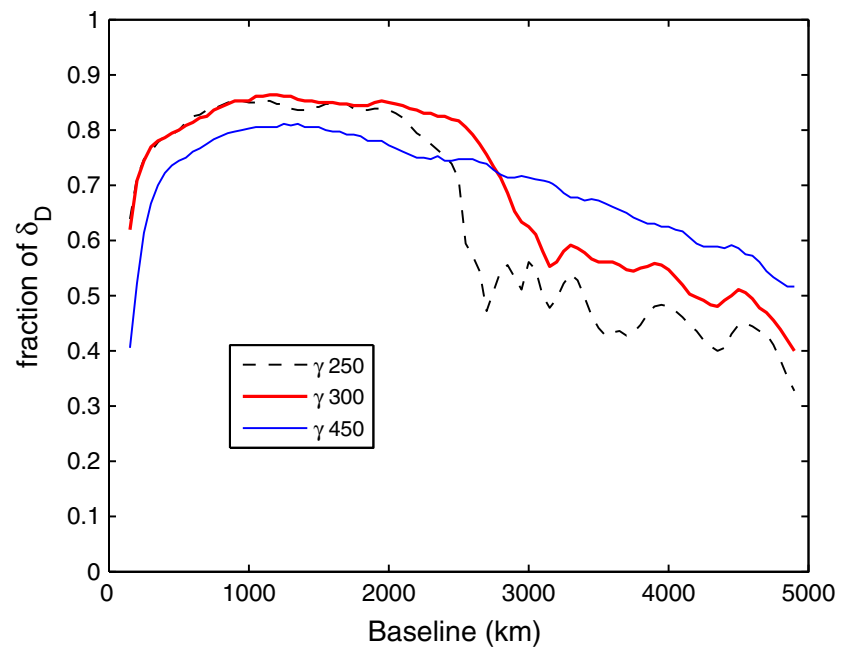

(c) Antineutrino beam, normal hierarchy

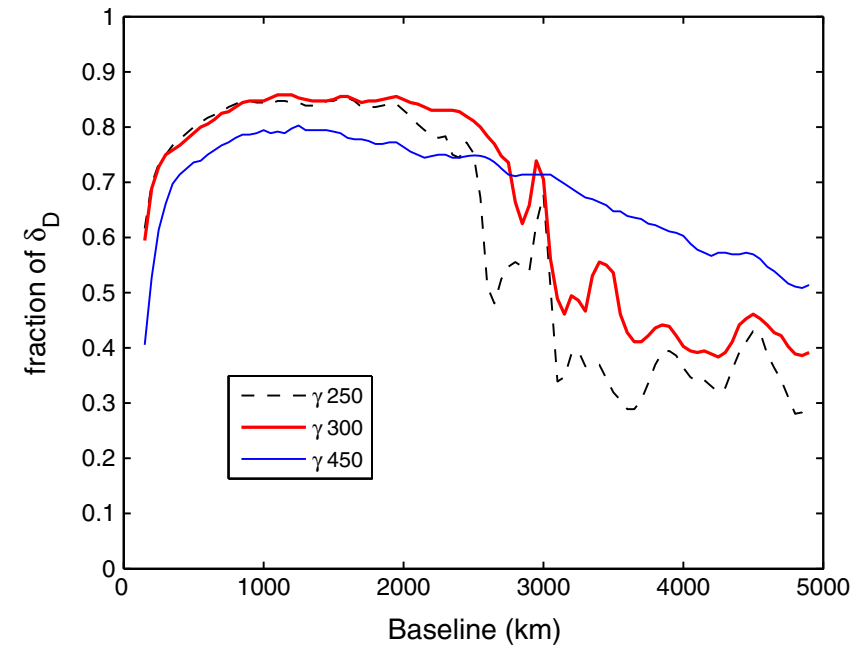

(b) Neutrino beam, inverted hierarchy

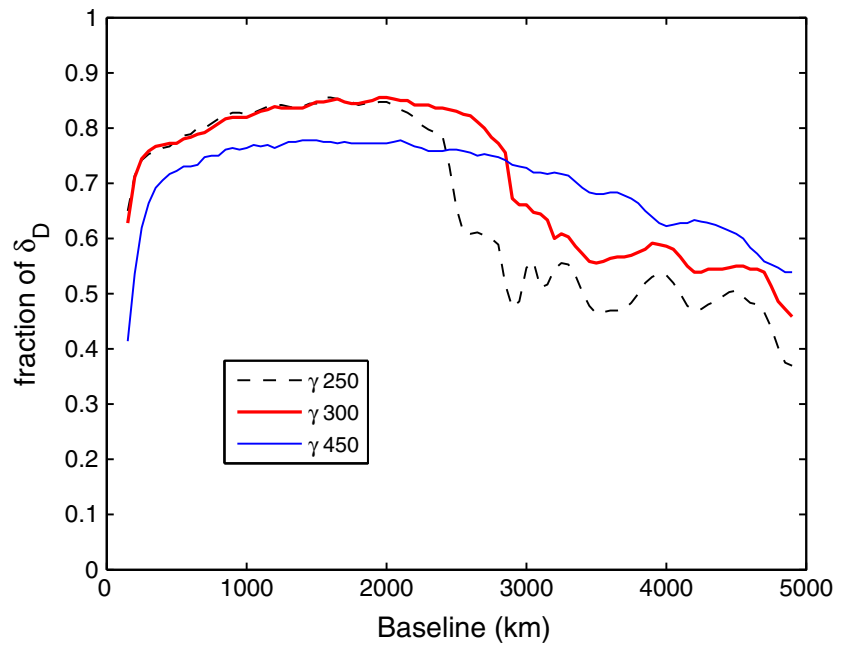

(d) Antineutrino beam, inverted hierarchy

Fig. 2 The fraction of the $\delta_{D}$ parameter for which $C P$ can be established at higher than $95 \%$ CL after 4 years of data taking versus baseline. The rest of description is as in Fig. 1

For $L=1300 \mathrm{~km}$, (corresponding to the baseline of the LBNE setup from the FermiLAB to Sanford underground research facility in South Dakota [57]), we also observe that $\gamma=300$ with WC detector is promising and can outperform the $\gamma=450$ setup with TASD detector. Figure 5 shows $\Delta \delta_{D}$ versus $\gamma$ for $L=1300 \mathrm{~km}$ and $L=2300 \mathrm{~km}$. The latter corresponds to the baseline for the LBNO setup from CERN to Finland [58]. The plots show that the setup with $\gamma=200-300$ and $L=1300 \mathrm{~km}$ can measure $\delta_{D}=90^{\circ}$ with a remarkable precision and also have an outstanding coverage of the $\delta_{D}$ range. At this baseline, increasing $\gamma$ from 200 to 300 does not much improve the sensitivity to $\delta_{D}$.

For relatively short baselines $L \sim 100 \mathrm{~km}, \sin \left(\lambda_{2}-\right.$ $\left.\lambda_{1}\right) / 2 \ll 1$, so the contributions of the first terms in Eqs. (1) and (2) are subdominant relative to the second terms. As a result, the interference between the first and second terms which is the only contribution sensitive to $\delta_{D}$ will be suppressed; i.e., when $\delta_{D}$ varies between 0 and $\pi$ the variation of $P_{e \mu}$ and $P_{\bar{e}} \bar{\mu}$ will be of order of $\sin \left(\lambda_{2}-\lambda_{1}\right) / 2 \sim$ $0.05 L /(130 \mathrm{~km})$. On the other hand, for $L>1000 \mathrm{~km}$, $\sin \left(\lambda_{2}-\lambda_{1}\right) / 2 \sim 1$ and the two terms in Eqs. (1) and (2) are of the same order, making the variation of the oscillation probabilities due to the variation of $\delta_{D}$ of order of the oscillation probabilities themselves. As a result, deriving $\delta_{D}$ from a $130 \mathrm{~km}$ setup such as CERN to Frejus requires a different strategy than that of a very long baseline setup with $L>1000 \mathrm{~km}$. This is demonstrated in Figs. 5 and 6. From Fig. 5 we observe that the pure $\bar{\nu}_{\mathrm{e}}$ run in the case of the setup with $L=1300 \mathrm{~km}$ has a better prospect but as seen in Fig. 6, in the case of the $L=130 \mathrm{~km}$ baseline a mixed run of neutrino and antineutrino can perform better than pure $\nu_{\mathrm{e}}$ or $\bar{\nu}_{\mathrm{e}}$ runs. For $\sin \left(\lambda_{2}-\lambda_{1}\right) / 2 \ll 1$, the uncertainties in 


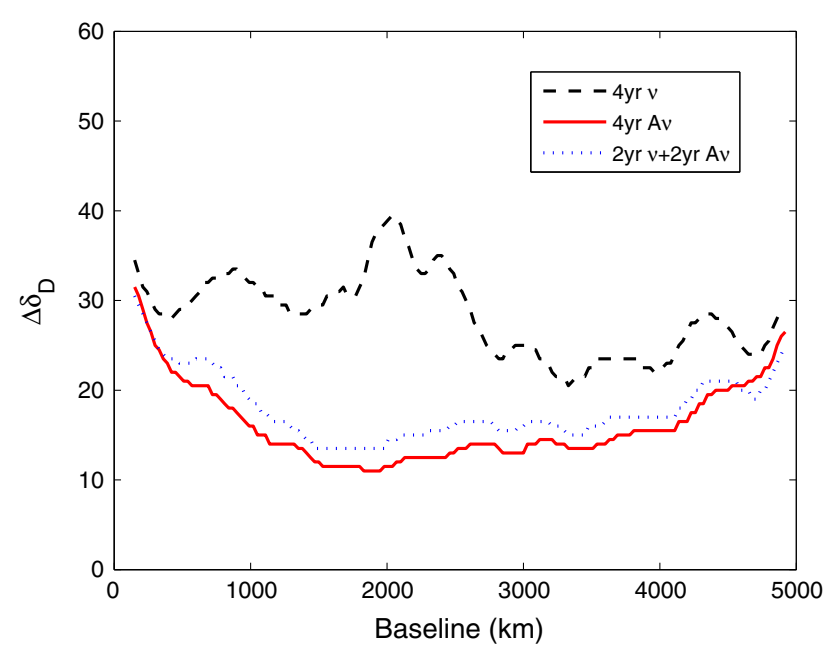

Fig. 3 Uncertainty within which $\delta_{D}=90^{\circ}$ can be measured versus baseline at $1 \sigma \mathrm{CL}$. The neutrino parameters are as in Fig. 1. The hierarchy is taken to be normal. For the neutrino and antineutrino beams, $2.2 \times 10^{18}$ and $5.8 \times 10^{18}$ decays per year are assumed, respectively. The curves shown with the dashed and solid lines, respectively, show the results of 4 years run in neutrino mode from the ${ }^{18} \mathrm{Ne}$ decay and 4 years run in antineutrino mode from the ${ }^{6} \mathrm{He}$ decay. The curve shown by dotted line displays the results of 2 years of neutrino run combined with 2 years of antineutrino run. The boost factors of the beams are taken to be 300

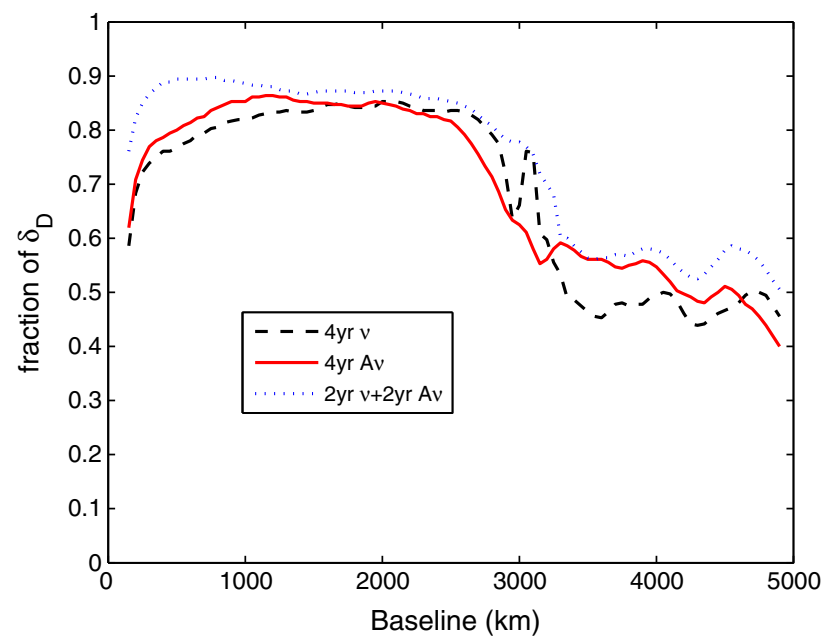

Fig. 4 The fraction of the $\delta_{D}$ parameter for which $C P$ can be established at higher than $95 \%$ CL versus baseline. The rest of the description is as that of Fig. 3

neutrino parameters (especially the uncertainties of $\theta_{13}$ and $\theta_{23}$ ) induce a significant uncertainty in the derivation of $\delta_{D}$. If we turn off the error in these parameters, the performance of the CERN to Frejus setup will be competitive with that of the LBNE setup, but considering the realistic uncertainty in these parameters as outlined in the previous section, the sensitivity of the LBNE setup to $\delta_{D}$ is much better than the $L=130 \mathrm{~km}$ setup. This can be confirmed by comparing Figs. 5 and 6.
For the $130 \mathrm{~km}$ setup, the oscillation probabilities can be approximately written as $P_{\bar{e} \bar{\mu}} \simeq \mid i s_{12}^{m} c_{23} \sin \left(\lambda_{2}-\lambda_{1}\right)+$ $\left.s_{13}^{m} s_{23} \mathrm{e}^{i \delta_{D}}\left(\mathrm{e}^{i \lambda_{3}}-1\right)\right|^{2}$ and $P_{e \mu} \simeq \mid i c_{12}^{m} c_{23} \sin \left(\lambda_{2}-\lambda_{1}\right)+$ $\left.s_{13}^{m} s_{23} \mathrm{e}^{-i \delta_{D}}\left(\mathrm{e}^{i \lambda_{3}}-1\right)\right|^{2}$. Since we are far from the 31 resonance, $s_{13}^{m}$ is not very different from $s_{13}$ and is approximately the same for normal and inverted hierarchies. As a result, replacing $\delta_{D} \rightarrow \pi-\delta_{D}$ and $\Delta m_{13}^{2} \rightarrow-\Delta m_{13}^{2}$ (i.e., $\lambda_{3} \rightarrow-\lambda_{3}$ ), the oscillation probability does not change. That is why in Fig. 6 the $\Delta \delta_{D}$ plots for normal and inverted hierarchies are practically the same. If we take a value other than $90^{\circ}$ as the true value of $\delta_{D}$, we will not have such a symmetry. However, as seen in Figs. 5 and 6, the general behavior for normal and inverted hierarchies are similar. With the present SPS setup, CERN cannot enhance $\gamma$ over 150 for the ${ }^{6} \mathrm{He}$ ions [29]. On the other hand, from Fig. 6, we observe that with $L=130 \mathrm{~km}$, there is no point in seeking higher values of $\gamma$. In fact, at $\gamma=150$, the fraction of CP-violating $\delta_{D}$ parameter for which CP-violation can be established is slightly higher than that for $\gamma>250$.

From comparing Figs. 5 and 6, we observe that the best performance can be achieved by an $L=1300 \mathrm{~km}$ setup and antineutrino run. For example, while with the CERN to Frejus setup, $\delta_{D}=90^{\circ}$ can be measured with only an uncertainty of $\Delta \delta_{D}=35^{\circ}$, with a $1,300 \mathrm{~km}$ setup, the uncertainty can be lowered down to $\Delta \delta_{D}=15^{\circ}$. Notice that for these setups, the same detector (500 kton WC) is assumed. Although with longer baselines the flux decreases, instead $\lambda_{2}-\lambda_{1}$ in Eq. (2) becomes larger, so a moderate precision in the $P_{\bar{e} \bar{\mu}}$ measurement will suffice to extract $\delta_{D}$. For measuring $\delta_{D}=90^{\circ}$, the $L=2300 \mathrm{~km}$ setup with the $\bar{v}_{\mathrm{e}}$ run seems to be competitive with the $L=1300 \mathrm{~km}$ setup; however, the fraction of $\delta_{D}$ to be established by the $L=1300 \mathrm{~km}$ setup is considerably higher. Among the setups that we have considered the $L=1300 \mathrm{~km}$ setup with a $500 \mathrm{kton} \mathrm{WC}$ and the $\bar{v}$ run seems to be the most promising one. In Fig. 5, we observe that for $200<\gamma<300$, the curves corresponding to the $\bar{\nu}_{\mathrm{e}}$ run are almost flat.

As expected for $L>1000 \mathrm{~km}$, the results are highly sensitive to the central values of $\Delta m_{31}^{2}$. The oscillatory behavior in Fig. 1 that we discussed before implies such a sensitivity. In fact, the setup that we are proposing can simultaneously extract $\delta_{D}$ and $\Delta m_{31}^{2}$. Figure 7 shows 68 and $95 \%$ CL contours for $\gamma=300$ and $L=1300 \mathrm{~km}$ (LBNE). In drawing these plots, the hierarchy is assumed to be known; however, the measured value of $\Delta m_{31}^{2}$ is not used. The precision in $\Delta m_{31}^{2}$ can drastically be improved by forthcoming experiments. In [41], it is shown that combining the T2K and PINGU results, $0.7 \%$ precision in $\Delta m_{31}^{2}$ is achievable. In Fig. 7 the vertical lines show the $0.7 \%$ uncertainty in $\Delta m_{31}^{2}$ around the 'true' value of $\Delta m_{31}^{2}$. As seen from the figure, for the case of the antineutrino beam the uncertainty in $\Delta m_{31}^{2}$ will not significantly increase the uncertainty in the $\delta_{D}$ determination. 


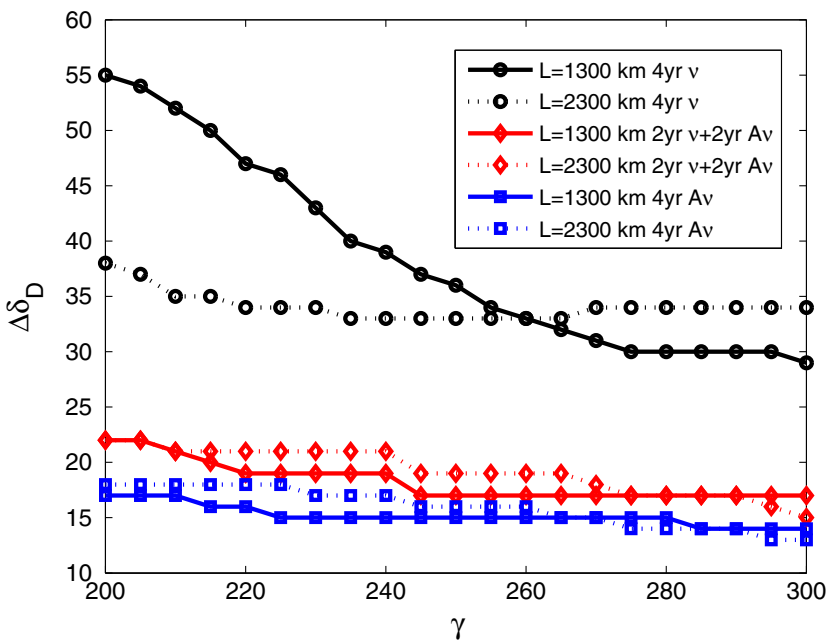

(a) Normal hierarchy

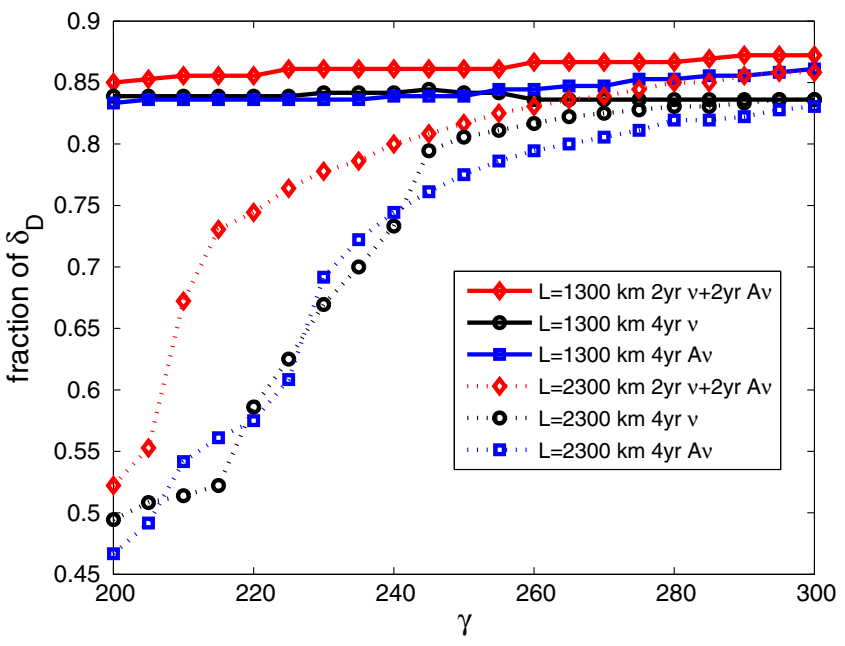

(c) Normal hierarchy

Fig. 5 The CP-discovery potential by the setups with baselines equal to 2,300 and $1,300 \mathrm{~km}$ after 4 years of data taking versus the boost factors of the neutrino and antineutrino beams. In the left (right) panels, the hierarchy is taken to be normal (inverted). Upper panels the uncertainty

\section{Conclusions}

Measuring the CP-violating phase by a beta beam facility has been extensively studied in the literature. Most of the recent studies have focused on relatively high energy beams with $\gamma>300$. The reason is that for a given baseline, the number of detected neutrinos increases approximately as $\gamma^{3}$. However, for lower energy beta beam, a large volume WC detectors [30] can be employed that can compensate for the decrease of flux and cross section. Moreover, with the relatively large value of $\theta_{13}$ chosen by Nature, having enough statistics will not be the most serious challenge for measuring the $\mathrm{CP}$-violating phase. Considering these facts, we have explored the CP-discovery reach of an intermediate

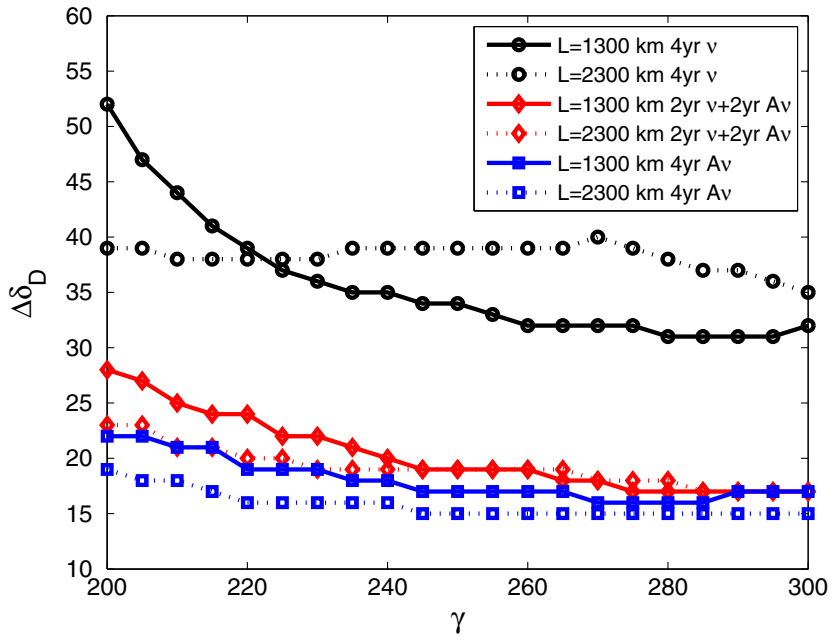

(b) Inverted hierarchy

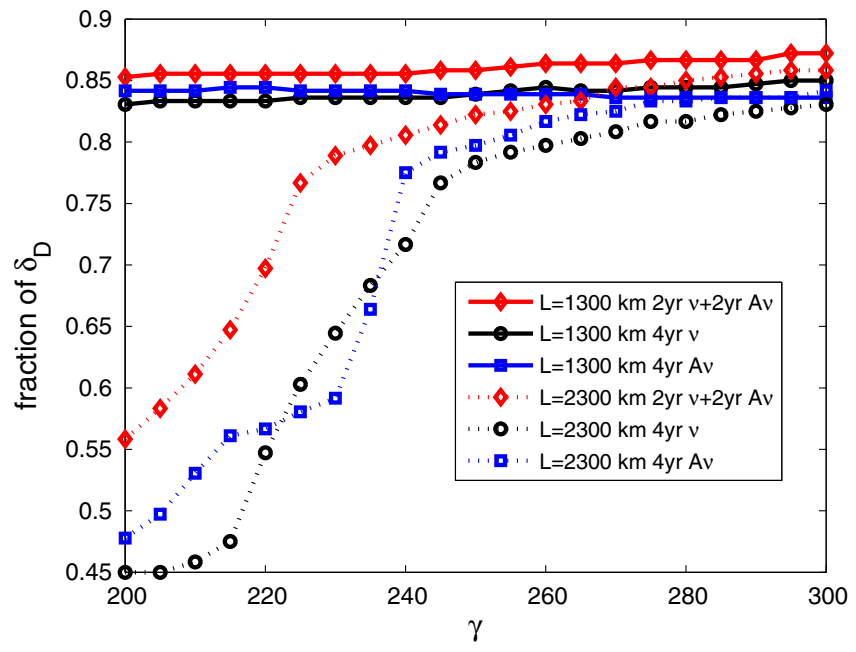

(d) Inverted hierarchy

within which $\delta_{D}=90^{\circ}$ can be measured at $1 \sigma \mathrm{CL}$ versus the boost factors of $v_{\mathrm{e}}$ and $\bar{v}_{\mathrm{e}}$ beams from the ${ }^{18} \mathrm{Ne}$ and ${ }^{6} \mathrm{He}$ decay. Lower panels the fraction of the $\delta_{D}$ parameter for which $C P$ can be established at higher than $95 \% \mathrm{CL}$

energy beta beam for various baselines and different neutrino vs. antineutrino combinations using the GLoBES software [32,33]. We have discussed the precision with which $\delta_{D}$ can be measured, assuming that by the time that the required facilities are ready the hierarchy is also determined. Our results do not depend much on which mass ordering is chosen.

We have found that a setup with only an antineutrino run with $200<\gamma<300$ and a baseline of $L=1,300 \mathrm{~km}$ has an excellent discovery potential. Four years run of such a setup with $5.8 \times 10^{18}{ }^{6} \mathrm{He}$ decays per year can establish CPviolation at $95 \% \mathrm{CL}$ for more than $85 \%$ of the $\delta_{D}$ parameter range. If $\delta_{D}=90^{\circ}$, this setup can determine it with impressive precision $\delta_{D}=90^{\circ} \pm 8^{\circ}$ for an inverted hierarchy and 


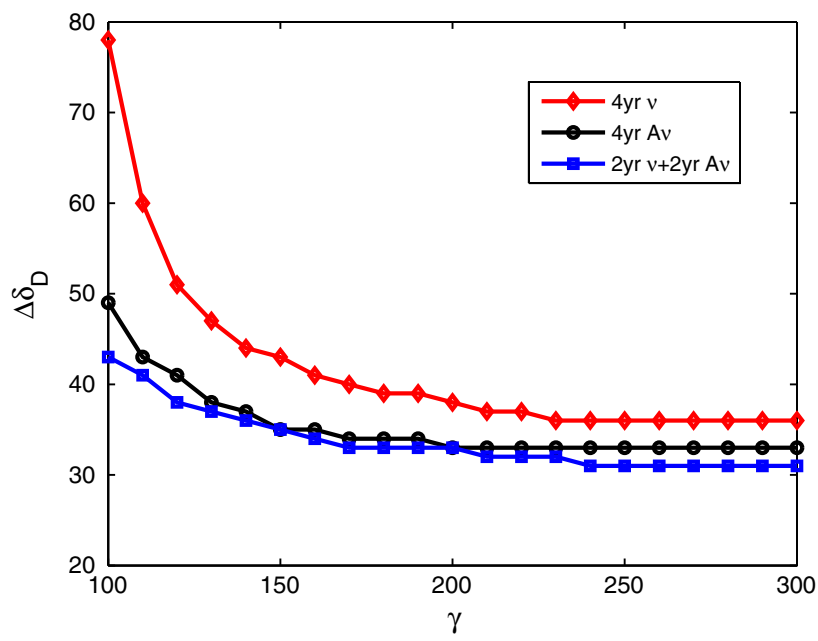

(a) Normal hierarchy

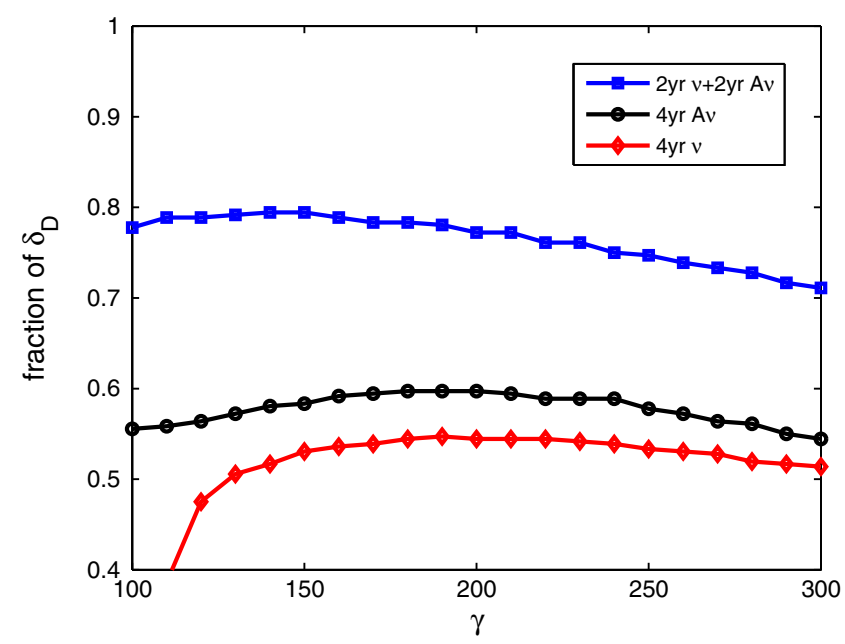

(c) Normal hierarchy

Fig. 6 The CP-discovery potential of a $130 \mathrm{~km}$ experiment (distance between CERN to Frejus) after 4 years of data taking versus the boost factors of neutrino and antineutrino beams. In the left (right) panels, the hierarchy is taken to be normal (inverted). Upper panels the uncertainty

$\delta_{D}=90^{\circ} \pm 7^{\circ}$ for a normal hierarchy at $1 \sigma \mathrm{CL}$. Such a baseline corresponds to the distance between FermiLAB to Sanford underground research facility in South Dakota. A baseline of $L=1300 \mathrm{~km}$ seems to be close to the optimal distance to measure the Dirac CP-violating phase. We have found that for this baseline a setup with intermediate values of $\gamma$ in the range $200<\gamma<300$ with a 500 kton WC detector can outperform that with $\gamma=450$ and 50 kton TASD.

For very long baselines with $L>1000 \mathrm{~km}$, a pure antineutrino source from ${ }^{6} \mathrm{He}$ enjoys a better performance than a mixed neutrino antineutrino run. On the other hand for shorter baselines, a balanced neutrino-antineutrino mode gives better results. We have specifically discussed the CERN to Frejus setup with $L=130 \mathrm{~km}$ baseline. We have found that

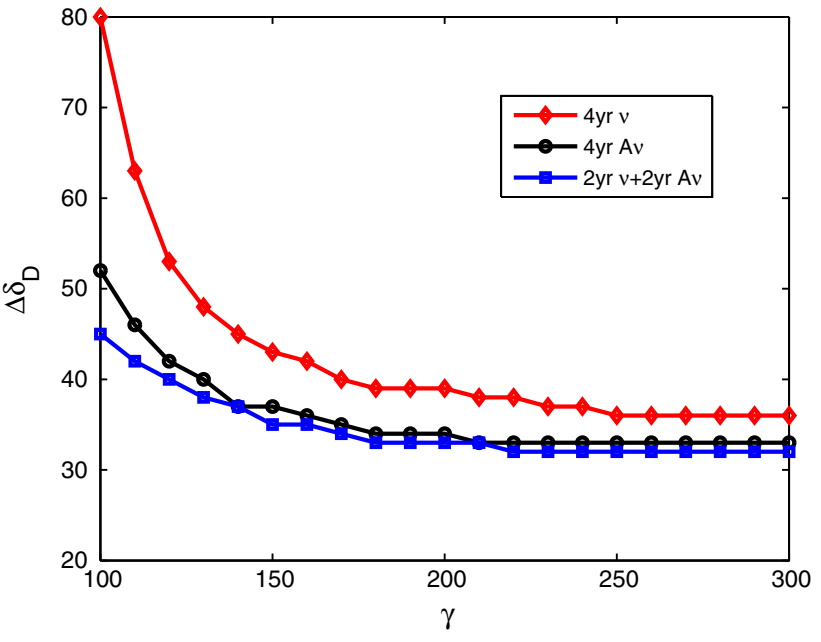

(b) Inverted hierarchy

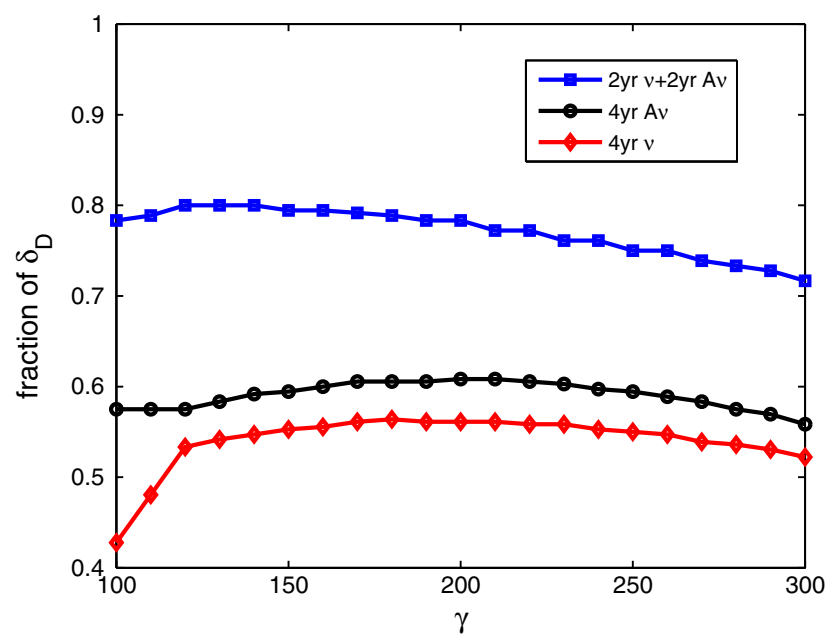

(d) Inverted hierarchy

within which $\delta_{D}=90^{\circ}$ can be measured at $1 \sigma \mathrm{CL}$ versus the boost factors of the $v_{\mathrm{e}}$ and $\bar{v}_{\mathrm{e}}$ beams from the ${ }^{18} \mathrm{Ne}$ and ${ }^{6} \mathrm{He}$ decay. Lower panels the fraction of the $\delta_{D}$ parameter for which $C P$ can be established at higher than $95 \% \mathrm{CL}$

with 2 years of neutrino mode from $2.2 \times 10^{18}$ decays of ${ }^{18} \mathrm{Ne}$ per year combined with 2 years of antineutrino mode from $5.8 \times 10^{18}$ decays of ${ }^{6} \mathrm{He}$ per year both with $\gamma=150$ (the largest boost that can be obtained for ${ }^{6} \mathrm{He}$ with the present SPS accelerator at CERN [29]), the CP-violation can be established for about $80 \%$ of the $\delta_{D}$ parameter range. With such a setup and runtime, if the true value of $\delta_{D}$ is equal to $90^{\circ}$, it can be measured as $\delta_{D}=90^{\circ} \pm 18^{\circ}$ at $1 \sigma$ CL. By increasing $\gamma$ to higher values, the precision in the $\delta_{D}$ measurements slightly improves, however, still with a similar detector and antineutrino run, the performance of $L=1,300 \mathrm{~km}$ can be better.

Our conclusion is that a beta beam facility with $200<$ $\gamma<300$, a baseline of $L \simeq 1300 \mathrm{~km}$, and 500 kton WC run- 


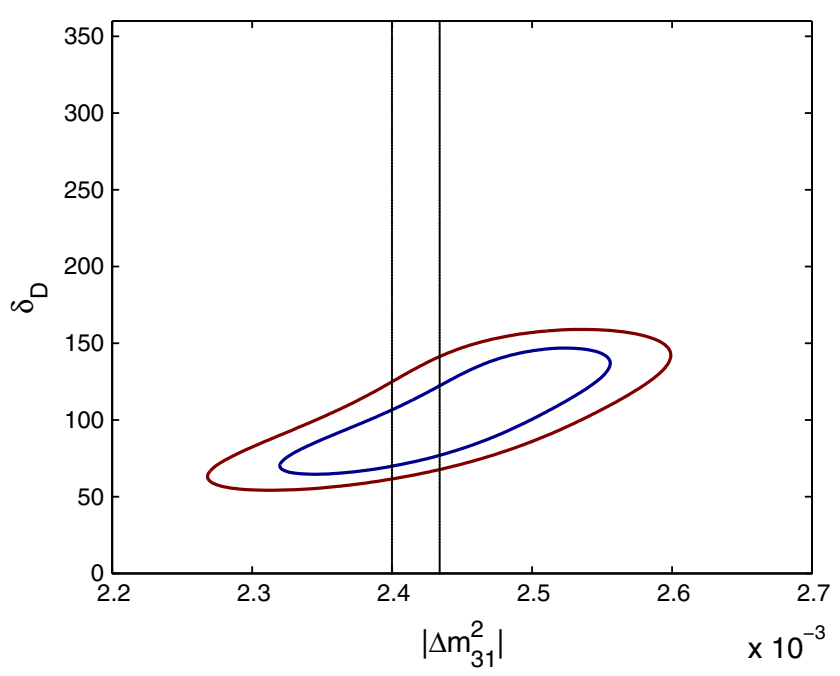

(a) Neutrino beam, normal hierarchy

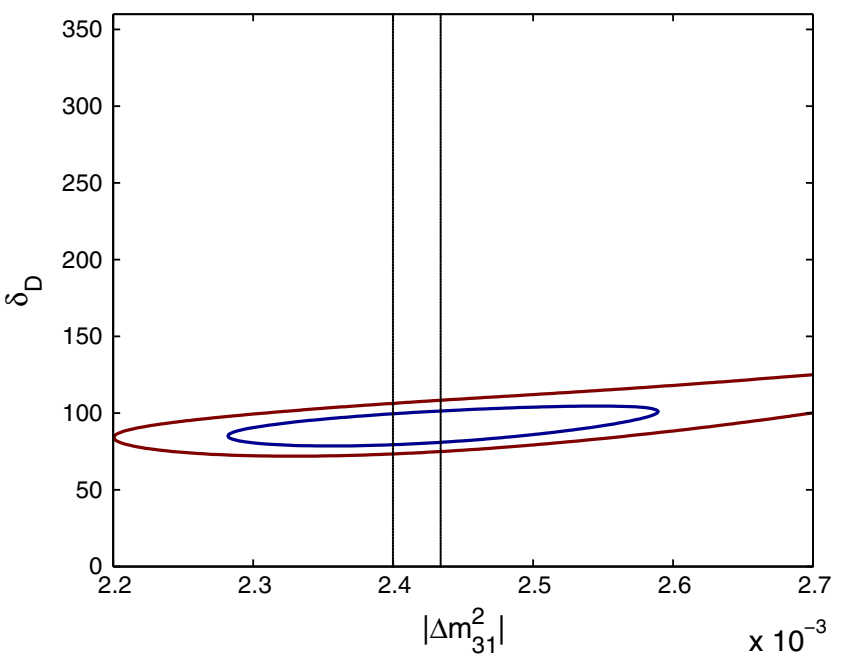

(c) Antineutrino beam, normal hierarchy

Fig. 7 Determination of $\delta_{D}$ and $\Delta m_{31}^{2}$ with $\gamma=300$ and $L=1300 \mathrm{~km}$ after 4 years of data taking. The contours show $68 \% \mathrm{CL}$ and $95 \%$ $\mathrm{CL}$. In the upper (lower) panels, a neutrino (antineutrino) beam with $2.2 \times 10^{18}\left(5.8 \times 10^{18}\right)$ decays per year is assumed. In the left (right) panels, the hierarchy is taken to be normal (inverted). The true value

ning in the antineutrino mode from ${ }^{6} \mathrm{He}$ decay is an optimal option for establishing CP-violation in the lepton sector and the measurement of $\delta_{D}$. The location of source and detector might be, respectively, FermiLAB and Sanford underground laboratory in South Dakota.

Acknowledgments The authors would like to thank A. Yu. Smirnov for encouragement and very useful comments. They also thank F. Terranova and W. Winter for fruitful comments. P. B. acknowledges H. Mosadeq for technical help in running the computer codes. They also acknowledges partial support from the European Union FP7 ITN INVISIBLES (Marie Curie Actions, PITN-GA-2011-289442). Y.F. thanks the staff of Izmir technical institute (IZTECH) where a part of this work was carried out for generous support and hospitality. The authors also thank the anonymous referee for useful remarks.

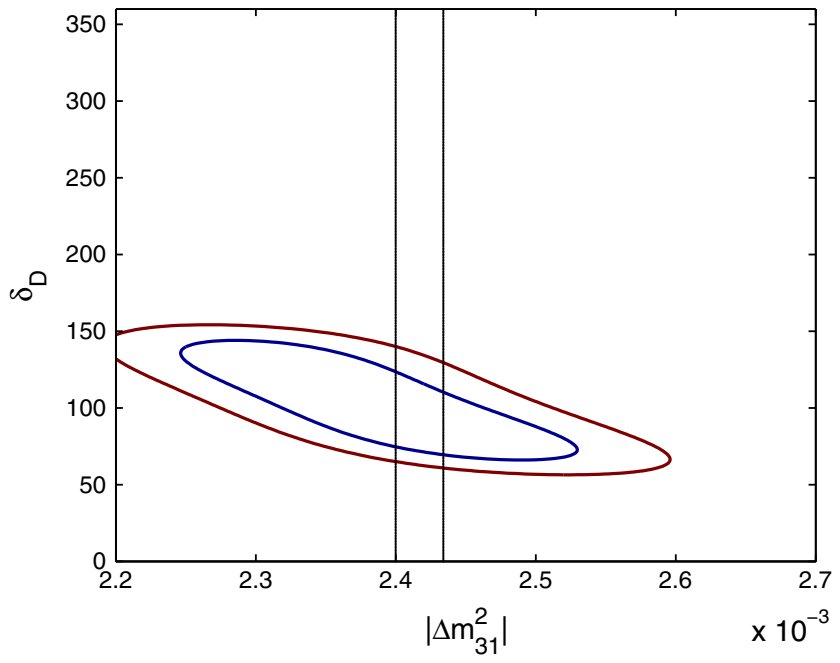

(b) Neutrino beam, inverted hierarchy

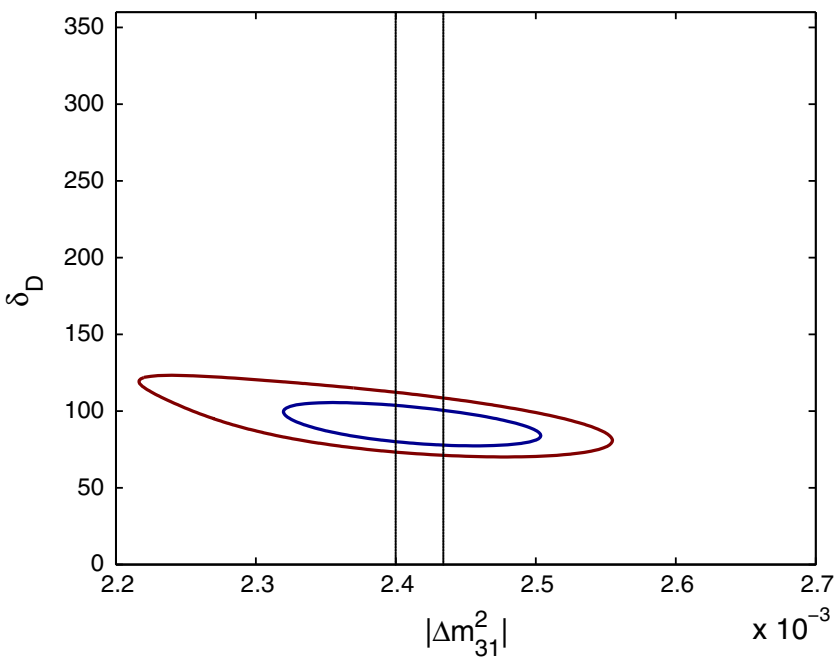

(d) Antineutrino beam, inverted hierarchy

of $\delta_{D}$ is taken to be $90^{\circ}$. For a normal (inverted) hierarchy, we take $\Delta m_{31}^{2}=2.421 \times 10^{-3} \mathrm{eV}^{2}\left(\Delta m_{31}^{2}=-2.35 \times 10^{-3} \mathrm{eV}^{2}\right)$. The vertical lines show a $0.7 \%$ uncertainty in $\Delta m_{31}^{2}$ (e.g., for the normal hierarchy $\left.\Delta m_{31}^{2}=2.421 \times 10^{-3} \mathrm{eV}^{2} \times(1 \pm 0.7 \%) \mathrm{eV}^{2}\right)$

Open Access This article is distributed under the terms of the Creative Commons Attribution License which permits any use, distribution, and reproduction in any medium, provided the original author(s) and the source are credited.

Funded by $\mathrm{SCOAP}^{3}$ / License Version CC BY 4.0.

\section{References}

1. F.P. An et al. [DAYA-BAY Collaboration], Phys. Rev. Lett. 108, 171803 (2012). arXiv: 1203.1669 [hep-ex]

2. J.K. Ahn et al. [RENO Collaboration], Phys. Rev. Lett. 108, 191802 (2012). arXiv:1204.0626 [hep-ex]

3. Y. Abe et al. [DOUBLE-CHOOZ Collaboration], Phys. Rev. Lett. 108, 131801 (2012). arXiv:1112.6353 [hep-ex] 
4. A.Bandyopadhyay et al. [ISS Physics Working Group Collaboration], Rept. Prog. Phys. 72, 106201 (2009). arXiv:0710.4947 [hep$\mathrm{ph}]$

5. M.V. Diwan, D. Beavis, M.-C. Chen, J. Gallardo, S. Kahn, H. Kirk, W. Marciano, W. Morse et al., Phys. Rev. D 68, 012002 (2003). hep-ph/0303081

6. Y. Farzan, A.Y. Smirnov, Phys. Rev. D 65, 113001 (2002). hep-ph/ 0201105

7. H. Zhang, Z.-z. Xing, Eur. Phys. J. C 41, 143 (2005). hep-ph/ 0411183

8. A. Bandyopadhyay et al. [ISS Physics Working Group Collaboration], Rept. Prog. Phys. 72, 106201 (2009). arXiv:0710.4947 [hep-ph]

9. G. Ahuja, M. Gupta, Phys. Rev. D 77, 057301 (2008). hep-ph/ 0702129 [HEP-PH]

10. Z.-z. Xing, H. Zhang, Phys. Lett. B 618, 131 (2005). hep-ph/ 0503118

11. S. Antusch, S.F. King, C. Luhn, M. Spinrath, Nucl. Phys. B 850, 477 (2011). arXiv:1103.5930 [hep-ph]

12. A. Dueck, S. Petcov, W. Rodejohann, Phys. Rev. D 82, 013005 (2010). arXiv:1006.0227 [hep-ph]

13. Z.-z. Xing, S. Zhou, Phys. Lett. B 666, 166 (2008). arXiv:0804. 3512 [hep-ph]

14. P. Zucchelli, Phys. Lett. B 532, 166 (2002)

15. C. Volpe, in Proceedings of 13th Lomonosov Conference on Elementary Particles: Particle Physics on the Eve of the LHC, Moscow, 2007, pp. 146-153. arXiv:0802.3352 [hep-ph]

16. C. Volpe, Prog. Part. Nucl. Phys. 64, 325 (2010). arXiv:0911.4314 [hep-ph]

17. C. Orme, JHEP 1007, 049 (2010). arXiv:0912.2676 [hep-ph]

18. P. Coloma, A. Donini, P. Migliozzi, L. Scotto Lavina, F. Terranova, Eur. Phys. J. C 71, 1674 (2011). arXiv:1004.3773 [hep-ph]

19. J. Bernabeu, C. Espinoza, C. Orme, S. Palomares-Ruiz, S. Pascoli, AIP Conf. Proc. 1222, 174 (2010)

20. S.K. Agarwalla, A. Raychaudhuri, A. Samanta, Phys. Lett. B 629, 33 (2005). hep-ph/0505015

21. S.K. Agarwalla, S. Choubey, A. Raychaudhuri, Nucl. Phys. B 798, 124 (2008). arXiv:0711.1459 [hep-ph]

22. S.K. Agarwalla, P. Huber, Phys. Lett. B 693, 114 (2010). arXiv: 0909.2257 [hep-ph]

23. D. Meloni, O. Mena, C. Orme, S. Palomares-Ruiz, S. Pascoli, JHEP 0807, 115 (2008). arXiv:0802.0255 [hep-ph]

24. D. Meloni, O. Mena, C. Orme, S. Pascoli, S. Palomares-Ruiz, PoS NUFACT 08, 133 (2008)

25. S.K. Agarwalla, Y. Kao, T. Takeuchi, arXiv:1302.6773 [hep-ph]

26. M. Blennow, A.Y. Smirnov, Adv. High Energy Phys. 2013, 972485 (2013). arXiv:1306.2903 [hep-ph]

27. T.R. Edgecock, O. Caretta, T. Davenne, C. Densham, M. Fitton, D. Kelliher, P. Loveridge, S. Machida et al., Phys. Rev. ST Accel. Beams 16, 021002 (2013). arXiv:1305.4067 [physics.acc-ph]

28. P. Huber, M. Lindner, M. Rolinec, W. Winter, Phys. Rev. D 73, 053002 (2006). hep-ph/0506237

29. M. Mezzetto, J. Phys. G 29, 1771 (2003). hep-ex/0302007

30. W. Winter, Phys. Rev. D 78, 037101 (2008). arXiv:0804.4000 [hep$\mathrm{ph}]$

31. S.K. Agarwalla, S. Choubey, A. Raychaudhuri, W. Winter, JHEP 0806, 090 (2008). arXiv:0802.3621 [hep-ex]
32. P. Huber, M. Lindner, W. Winter, Comput. Phys. Commun. 167, 195 (2005). hep-ph/0407333

33. P. Huber, J. Kopp, M. Lindner, M. Rolinec, W. Winter, Comput. Phys. Commun. 177, 432 (2007). hep-ph/0701187

34. M.C. Gonzalez-Garcia, M. Maltoni, J. Salvado, T. Schwetz, JHEP 1212, 123 (2012). arXiv:1209.3023 [hep-ph] (see also, v1.1 results in http://www.nu-fit.org)

35. E.K. Akhmedov, S. Razzaque, AYu. Smirnov, JHEP 02, 082 (2013) arXiv:1205.7071 [hep-ph]

36. S.K. Agarwalla, T. Li, O. Mena, S. Palomares-Ruiz, arXiv:1212. 2238 [hep-ph]

37. D. Franco et al., JHEP 1304, 008 (2013). arXiv:1301.4332 [hep-ex]

38. T. Ohlsson, H. Zhang, S. Zhou, Phys. Rev. D 88, 013001 (2013). arXiv:1303.6130 [hep-ph]

39. A. Esmaili, AYu. Smirnov, JHEP 1306, 026 (2013). arXiv:1304. 1042 [hep-ph]

40. M. Ribordy, A.Y. Smirnov, Phys. Rev. D 87, 113007 (2013). arXiv: 1303.0758 [hep-ph]

41. W. Winter, Phys. Rev. D 88, 013013 (2013). arXiv:1305.5539 [hep$\mathrm{ph}]$

42. M. Blennow, T. Schwetz, JHEP 1309, 089 (2013). arXiv:1306.3988 [hep-ph]

43. S.K. Agarwalla, S. Prakash, S.U. Sankar, JHEP 1307, 131 (2013). arXiv:1301.2574 [hep-ph]

44. F. Capozzi, E. Lisi, A. Marrone, arXiv:1309.1638 [hep-ph]

45. D.V. Forero, M. Tortola, J.W.F. Valle, Phys. Rev. D 86, 073012 (2012). arXiv:1205.4018 [hep-ph]

46. S.K. Raut, Mod. Phys. Lett. A 28, 1350093 (2013). arXiv:1209. 5658 [hep-ph]

47. A.M. Dziewonski, D.L. Anderson, Preliminary reference earth model. Phys. Earth Planet Inter. 25, 297 (1981)

48. F.F. Stancy, Physics of the Earth, 2nd edn. (Wiley, London, 1977)

49. A. Jansson, O. Mena, S.J. Parke, N. Saoulidou, Phys. Rev. D 78, 053002 (2008). arXiv:0711.1075 [hep-ph]

50. P. Huber, M. Lindner, W. Winter, Nucl. Phys. B 645, 3 (2002). hep-ph/0204352

51. L. Agostino et al. [MEMPHYS Collaboration], JCAP 1301, 024 (2013). arXiv:1206.6665 [hep-ex]

52. M.D. Messier, UMI-99-23965

53. E.A. Paschos, J.Y. Yu, Phys. Rev. D 65, 033002 (2002). hep-ph/ 0107261

54. A.A. Aguilar-Arevalo et al. [MiniBooNE Collaboration], Phys. Rev. D 88, 032001 (2013). arXiv: 1301.7067 [hep-ex]

55. P. Huber, M. Lindner, W. Winter, Comput. Phys. Commun. 167, 195 (2005). hep-ph/0407333

56. P. Huber, J. Kopp, M. Lindner, M. Rolinec, W. Winter, Comput. Phys. Commun. 177, 432 (2007). hep-ph/0701187

57. S. Childress, J. Strait, J. Phys. Conf. Ser. 408, 012007 (2013)

58. A. Stahl, C. Wiebusch, A.M. Guler, M. Kamiscioglu, R. Sever, A.U. Yilmazer, C. Gunes , D. Yilmaz et al., CERN-SPSC-2012-021 